\title{
Effect of Organo-mineral Fertilization on the Growth, Yield and Economic Profitability of Bean (Phaseolus vulgaris) in Eastern DRC
}

(Effet de la fertilisation organo- minérale sur la croissance, le rendement et la rentabilité économique de haricot (Phaseolus vulgaris) à l'Est de la RDC)

\author{
Chakirwa, Z.P ${ }^{1,2,3^{*}}$, Murhi, M.I. ${ }^{1,3}$, Aksanti, K.C ${ }^{2}$, Masirika, F.C. ${ }^{1}$, Banyanga, N.E ${ }^{1}$ et Lubobo, A.K. ${ }^{4}$ \\ Centre Interdisciplinaire pour le Développement Permanent (CIDEP) ${ }^{1}$ \\ Université Libre des Grands Lacs (ULGL) ${ }^{2}$ \\ Université Catholique de Bukavu (UCB) ${ }^{3}$ \\ CIAT/HarvestPlus
}

\begin{abstract}
In the face to low fertility existing in tropical soils, farmers in the Eastern Congo need cost-effective Soil Fertility Management (SFM) technologies to address the problem of low crop yields, which are particular lypronounced in grain legumes. This study assessed the financial returns associated with different SFM trials conducted on common bean grain in eastern Congo. Benefit-cost ratio analysis based on experimental data identified organic and mineral fertilizer technologies for common bean production. Two fields experiment were conducted at the Crop Research (INERA/Mulungu) and Walungu district (Ikoma). The experimental design was split plot with four replications. The main plot was the different time of application (2, 3 and 4weeks after sowing) and the sub plot were the organic and inorganic fertilizers (40 $\mathrm{N} \mathrm{kg} \mathrm{ha}^{-1}, 60 \mathrm{P}_{2} \mathrm{O}_{5}$ ha $^{-1}$ and $5 \mathrm{t}$ Fumier ha $\left.{ }^{-1}\right)$ compare to an absolute control $\left(0: 0 \mathrm{~N}: \mathrm{P} \mathrm{kg} \mathrm{ha}^{-1}\right.$ and $0 \mathrm{t}$ manure $\left.\mathrm{ha}^{-1}\right)$. The RWR21-54was the variety used. $\mathrm{N}$ was applied as urea, $\mathrm{P}$ was applied as triple superphosphate (TSP) and organic fertilizer was applied as cow manure. Plant height was significantly $(\mathrm{p}<0.05)$ influenced by $\mathrm{N}$ and $\mathrm{P}$ application in the two locations throughout the growing season. $\mathrm{N}, \mathrm{P}$ and manure application significantly $(\mathrm{p}<0.05)$ increased total dry matter (TDM, $\mathrm{kg} \mathrm{ha}^{-1}$ ), nodulation and leaf area (LA) of both locations in late vegetative and reproductive stages. Kabare site consistently had higher TDM and LA compared to Walungusite throughout the growing season. Average seed yield in fertilized plot was 2 times higher than the control(averaged over all N, P and manure levels) in both experiments. The highest seed yield was obtained with 40:60 N:P $\mathrm{P}_{2} \mathrm{O}_{5} \mathrm{~kg} \mathrm{ha}^{-1}$ and $5 \mathrm{t} \mathrm{ha}^{-1}$ fertilization $\left(1781.23 \mathrm{~kg} \mathrm{ha}^{-1}\right.$ «Kabare» and $1641.39 \mathrm{~kg} \mathrm{ha}^{-1}$ «Walungu») which was not economic dose. Significantly higher net returns (1637.50 ha ${ }^{1}$ «Kabare» and1641,67 $\mathrm{kg} \mathrm{ha}^{-1}$ «Walungu») and its benefits cost ratio (2.82 «Kabare» and 2.87 «Walungu») were alsoobtained with application of $60 \mathrm{P}_{2} \mathrm{O}_{5} \mathrm{~kg} \mathrm{ha}^{-1}$ in the both locations.
\end{abstract}

Key words: Nitrogen, phosphorus, manure, common bean and economic analysis

\section{Résumé}

Face au faible taux de fertilité de sol existants en Afrique Subtropicale, les agriculteurs de l'Est du Congo ont besoin de technologies de gestion de la fertilité du sol (GIFS) rentables pour résoudre le problème des faibles rendements des cultures, particulièrement élevés dans les légumineuses à grains. Cette étude a évalué les rendements financiers associés aux différents essais GIFS menés l'Est de la RDC sur la culture de Haricot afin d'examiner la décision d'adoption des agriculteurs et la volonté de payer pour les technologies les plus rentables financièrement. L'analyse du rapport bénéfice-coût basée sur des données expérimentales a été évalué pour les deux technologies adoptées (engrais organiques et minéraux) sur la croissance et le rendement du haricot. Pour y arriver, deux expérimentations ont été menées à Kabare (INERA/Mulungu) et à Walungu (Ikoma centre). Le dispositif expérimental était celui des parcelles divisées (split plot) comportant quatre répétitions. Le facteur secondaire était les différentes de temps d'application (2, 3 et 4 
semaines après le semis) et le facteur principal étaient les différentes options de la gestion de fertilité des sols $\left(40 \mathrm{~N} \mathrm{~kg} \mathrm{ha}^{-1}, 60 \mathrm{P}_{2} \mathrm{O}_{5}\right.$ ha $^{-1}$ et $\left.5 \mathrm{t}_{\text {Fumier ha }}{ }^{-1}\right)$ comparé au témoin $\left(0: 0 \mathrm{~N}: \mathrm{P} \mathrm{kg} \mathrm{ha}^{-1}\right.$ et $0 \mathrm{t}$ de fumier ha $\left.{ }^{1}\right)$. Le RWR21-54 était la variété utilisée. La source de l'azote $(\mathrm{N})$ était l'urée, celle de Phosphore $(\mathrm{P})$ était superphosphate triple (TSP) et enfin, celle de l'engrais organique était le fumier de vache. La hauteur de la plante a été significativement $(\mathrm{p}<0,05)$ influencé par l'application de $\mathrm{N}$ et $\mathrm{P}$ dans les deux sites expérimentaux. L'application de $\mathrm{N}, \mathrm{P}$ et de fumier augmente significativement $(\mathrm{p}<0,05)$ la matière sèche totale $\left(\mathrm{kg} \mathrm{ha}^{-1}\right)$, la nodulation et surface foliaire (ISF) durant les stades (végétatifs et reproductifs). Le site de Kabare a donné des meilleurs résultats par rapport à ceux de Walungu. Le rendement en grain ( $\mathrm{kg} / \mathrm{ha})$ dans la parcelle fertilisée était 2 fois plus élevé que le témoin (moyenné sur tous les niveaux de N, P et fumier) dans les deux sites expérimentaux. Le rendement en grain le plus élevé a été obtenu avec l'option GIFS 40$60 \mathrm{~N}$ : $\mathrm{P}_{2} \mathrm{O}_{5} \mathrm{~kg} \mathrm{ha}^{-1}$ et $5 \mathrm{t} \mathrm{ha}^{-1}$ fertilisation (1781.23 kg ha ${ }^{-1}$ «Kabare» and $1641.39 \mathrm{~kg} \mathrm{ha}^{-1}$ «Walungu»), mais n'étant pas une dose économique. Des rendements nets significativement plus élevés $\left(1637,50\right.$ ha $^{-1}$ « Kabare» and $1641,67 \mathrm{~kg} \mathrm{ha}^{-1}$ «Walungu») et leurs ratio bénéfice-coûts $(2,82$ «Kabare» and 2,87 «Walungu») ont été obtenus avec l'application de $60 \mathrm{P}_{2} \mathrm{O}_{5} \mathrm{~kg} \mathrm{ha}^{-1}$ dans les deux sites d'étude.

Mots-clés : Azote, phosphore, fumier, haricot commun et rentabilité économique

\section{Introduction}

Le haricot commun est riche en amidon, en protéines et en fibres alimentaires et c'est une excellente source de potassium, le sélénium, le molybdène, la thiamine, la vitamine B6 et l'acide folique (Maiti et Singh, 2007). Il est utilisé comme aliment et les gousses vertes non mûres sont cuites ou conservées comme légume (Brucher et al., 1977). Le haricot commun (Phaseolus vulgaris L.) avec d'autres légumineuses peut utiliser l'azote inorganique, l'azote provenant des engrais appliqués et l'azote fixé dans les nodosités des racines formées par les rhizobiums. Une combinaison de $\mathrm{N}$-sol et de $\mathrm{N}$ apporté sous-forme des engrais peut-être la meilleure solution pour un rendement maximal chez les légumineuses (Harper, 1974). La relation entre $\mathrm{N}$ et $\mathrm{P}$ est complexe et dépend de l'état du sol. Seulement 30 à $40 \%$ de l'engrais $\mathrm{N}$ appliqué par la méthode de diffusion conventionnelle est disponible pour la croissance des plantes ; le reste de l'azote est sujet à des pertes par volatilisation de l'ammoniac, dénitrification, lessivage, ruissellement et immobilisation biologique ou chimique (Craswell et al., 1981, Ladha et al., 2005).

L'azote et le phosphore ont été identifié comme l'un des principaux éléments nutritifs dans tropiques limitant la production des cultures (Groenland, 1982, Weiss, 1983, IRRI, 1990, Tisdale et al., 1990, Brady, 1990, ICRISAT, 1991). Dans les régions tropicales humides, l'azote est d'une grande importance en raison des fortes précipitations reçues dans cette région, accélérant la perte d'azote par lessivage. Dans les zones semiarides, la carence en azote résulte de la faible humidité du sol, ce qui entrave fortement le processus de minéralisation (Rowland, 1993). La plupart des sols tropicaux ont un faible échange cationique des fractions argileuses, souvent de faibles quantités de bases échangeables, une saturation en aluminium relativement élevée entraînant une fixation élevée du phosphore et une interférence entre les nutriments (Bennema, 1977). Les déficits d'azote et de phosphore sont un phénomène courant en RDC et peuvent être admirés par l'application d'engrais inorganiques et/ou de fumier (Nyandat, 1981 ; Allen et al., 1997).

Le haricot commun a un besoin élevé en azote $(\mathrm{N})$ pour exprimer son potentiel génétique. Cependant, le haricot a la capacité de fixer l'azote atmosphérique et l'utilisation en ce qui concerne la fertilité des sols et les besoins de la nutrition minérale, le phosphore est considéré comme le premier élément nutritif limitant le rendement de la culture de haricots sous les tropiques et l'azote vient en deuxième position. Ce travail s'est assigné d'examiner la croissance, la nodulation et le rendement ainsi que la rentabilité économique du haricot commun affecté les différentes options de la fertilité des sols (N, P et fumier).

\section{Materials and Methods}

\subsection{Description de la zone d'étude}

L'expérimentation a été réalisée dans deux sites dont à Kabare (INERA / Mulungu) se trouvant à $24 \mathrm{~km}$ de Bukavu, le chef-lieu du Sud-Kivu, situé sur une altitude de $1731 \mathrm{~m}$, avec des coordonnées géographiques de $28,47^{\circ} \mathrm{E}$ et $02,18^{\circ} \mathrm{N}$. Et le deuxième site était celui de Walungu (Ikoma) se trouvant sur une altitude de 1826 $\mathrm{m}$, ayant des coordonnées de $28,44^{\circ} \mathrm{E}$ et $02,34^{\circ} \mathrm{N}$. Les deux sites sont dans le climat tropical humide ayant sur une année deux saisons pluvieuses, la grande saison avec 5 mois des pluies de Septembre à Janvier et la 
petite saison de Février à Mai. Les précipitations annuelles sont comprises 1700-1800 m, la température annuelle de l'air est de 20,2 à $23,0^{\circ} \mathrm{C}$ et la température du sol est de $21,7-25,8^{\circ} \mathrm{C}$ à différentes profondeurs du sol (INERA, 2008).

\subsection{Dispositif expérimental and traitements}

Le dispositif expérimental était celui des parcelles divisées (split plot) comportant quatre répétitions. Le facteur secondaire était les différents temps d'application $(2,3$ et 4 semaines après le semis) et le facteur principal étaient les différentes options de la gestion de fertilité des sols $\left(40 \mathrm{~N} \mathrm{~kg} \mathrm{ha}^{-1}, 60 \mathrm{P}_{2} \mathrm{O}_{5}\right.$ ha $^{-1}$ et $5 \mathrm{t}$ Fumier ha $\left.{ }^{-1}\right)$ comparé au témoin $\left(0: 0 \mathrm{~N}\right.$ : $\mathrm{P} \mathrm{kg} \mathrm{ha}^{-1}$ et $0 \mathrm{t}$ de fumier ha $\left.{ }^{-1}\right)$. Le RWR21-54 était la variété utilisée. La source de l'azote $(\mathrm{N})$ était l'urée, celle de Phosphore (P) était superphosphate triple (TSP) et enfin, celle de l'engrais organique était le fumier de vache. L'unité expérimentale était de $2 \mathrm{~m} \times 2 \mathrm{~m}\left(4 \mathrm{~m}^{2}\right)$ ayant cinq lignes de semis, chacune contenant 10 plantes. Une distance de $1 \mathrm{~m}$ et 1,5 m a été laissée entre les parcelles principales et les blocs, respectivement. Trois graines étaient mises par poquet à une profondeur de plantation recommandée de $6 \mathrm{~cm}$ avec un écartement de $40 \mathrm{~cm}$ entre les lignes de semis et de $20 \mathrm{~cm}$ sur la ligne. Le démariage en deux plants par poquet a été effectué après deux semaines de semis. L'urée a été utilisé comme une source d'azote, le superphosphate triple (TSP) a été utilisé comme source de phosphore et le fumier est une source d'engrais organique. Les engrais ont été appliqués à différentes dates (2, 3 et 4 SAS) par poquet de semis. D'autres pratiques culturales pratiques agronomiques ont été maintenues uniformes pour tous les traitements recommandés dans les deux sites d'études.

\subsection{La collecte de l'échantillon de sol and l'analyse au laboratoire}

Un échantillon de sol a été collectée de 0 à $30 \mathrm{~cm}$ de profondeur, ont été recueillies à partir des points représentatifs du champ dans l'ensemble de deux sites d'expérimentation à l'aide de la méthode d'échantillonnage diagonale avant la plantation et l'échantillon composite a été obtenu. Le sol a été séché à l'air et rendu fin en utilisant un mortier et un pilon. Le sol a été passé à travers un tamis de $2 \mathrm{~mm}$ et l'analyse des propriétés physico-chimiques des sols ont été déterminées au laboratoire de l'Université Catholique de Bukavu (UCB), Bukavu, RDC, il s'agissait du pH, carbone, azote total, phosphore disponible, les cations échangeables $(\mathrm{Ca}, \mathrm{Mg}, \mathrm{K}, \mathrm{Na}, \mathrm{Al}+\mathrm{H}, \mathrm{Fe}, \mathrm{Zn})$ et les dimensions des particules ont été déterminé. Les échantillons analysés ont été déterminés suivant des normes pédologique du laboratoire. L'analyse de la taille des particules a été réalisée en utilisant la méthode de l'hydromètre qui dépend fondamentalement de la loi de Stokes (Boyoucos, 1962). Le pH du sol a été déterminé dans un rapport $1: 1$ (sol : eau) en utilisant un microprocesseur HI 9017pH-mètre. Le pH a été décrit selon Page et al. (1982). Le carbone organique du sol a été déterminé par la méthode modifiée de Walkley-Black décrit par Nelson et Sommers (1982). La teneur totale en azote des échantillons de sol a été déterminée par la digestion de Kjeldal et une procédure de distillation telle que décrite dans Soils Laboratory Staff (1984). Le phosphore disponible dans les échantillons de sol a été extrait avec la solution d'extraction $\mathrm{n}{ }^{\circ} 1$ de Bray comme décrit par Bray et Kurtz (1945). Détermination des cations échangeables bases échangeables (calcium, magnésium, potassium et sodium) dans le sol ont été déterminées dans un extrait d'acétate d'ammonium (NH4OAc) 1,0 M (Black, 1986) tandis que l'acidité échangeable (hydrogène et aluminium) a été déterminée dans un extrait de $\mathrm{KCl}$ 1,0 M (Page et al., 1982). La capacité d'échange cationique efficace a été obtenue par sommation des bases échangeables $\left(\mathrm{Ca}^{2+}, \mathrm{Mg}^{2+}, \mathrm{K}^{+}\right.$et $\left.\mathrm{Na}^{+}\right)$et acidité échangeable $\left(\mathrm{Al}^{3+}\right.$ et $\left.\mathrm{H}^{+}\right)$. Les résultats de l'analyse des sols dans les deux sites sont affichés dans le tableau 1.

\section{Tableau 1 : Caractéristiques du sol du site expérimental}

\begin{tabular}{|l|c|c|}
\hline Caractéristiques & Kabare (INERA/Mulungu) & Walungu (Ikoma) \\
\hline Paramètres chimiques & & \\
\hline $\mathrm{pH}$ & 6,04 & 5,30 \\
\hline $\mathrm{C}(\%)$ & 3,18 & 2,45 \\
\hline $\mathrm{N}(\%)$ & 0,26 & 0,16 \\
\hline $\mathrm{P}(\mathrm{ppm})$ & 34,52 & 26,18 \\
\hline $\mathrm{K}(\mathrm{méq} / 100 \mathrm{~g})$ & 0,79 & 0,35 \\
\hline $\mathrm{C} / \mathrm{N}$ & 13,17 & 15,31 \\
\hline $\mathrm{MO}(\%)$ & 5,49 & 4,22 \\
\hline
\end{tabular}




\begin{tabular}{|l|c|c|}
\hline CECE (méq/100g) & 18,13 & 4,83 \\
\hline $\mathrm{Ca}^{2+}(\mathrm{méq} / 100 \mathrm{~g})$ & 2,36 & 2,16 \\
\hline $\mathrm{Mg}^{2+}(\mathrm{méq} / 100 \mathrm{~g})$ & 0,77 & 0,67 \\
\hline $\mathrm{Fe}^{++}(\mathrm{ppm})$ & 159,34 & 189,54 \\
\hline $\mathrm{Zn}(\mathrm{ppm})$ & 130,80 & 110,80 \\
\hline Paramètres physiques & & \\
\hline Argile (\%) & 20,41 & 23,70 \\
\hline Limon (\%) & 26,81 & 38,90 \\
\hline Sable (\%) & 54,85 & 41,70 \\
\hline
\end{tabular}

Les résultats du tableau 1 montrent que le sol de Walungu (Ikoma) est de type ferrugineux tropical lessivé peu profond. Les caractéristiques physico-chimiques montrent que le sol est de texture sablo-limoneuse. Il est pauvre en argile. Le taux de MO était de 0,422 \%. La CEC varie en fonction des horizons, elle était de $4,83 \%$. Ce sol est acide avec un pH de 5,3. Pour le deuxième site expérimental (INERA/Mulungu), le sol est de type ferrugineux tropical lessivé profond. Les caractéristiques physico-chimiques montrent que les résultats suivent la même allure que sol de Walungu mais avec un taux supérieur d'amélioration. Le taux de MO est de 0,549\%. La CEC varie en fonction des horizons, elle est de 18,13\%. Ce sol est acide avec un pH de 6,04 .

Le tableau 2 montre la combinaison des traitements sur les deux sites expérimentaux

Tableau 2 : Combinaisons des traitements appliqués sur le terrain expérimental

\begin{tabular}{|c|c|}
\hline Traitements & Traitements détaillés \\
\hline $\mathrm{T}_{1} \mathrm{E}_{0}$ & 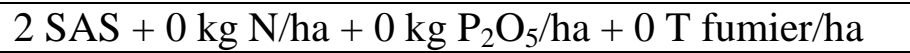 \\
\hline $\mathrm{T}_{1} \mathrm{E}_{1}$ & 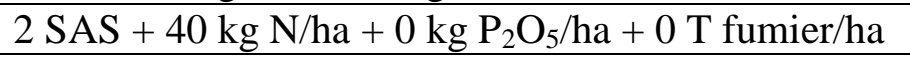 \\
\hline $\mathrm{T}_{1} \mathrm{E}_{2}$ & $2 \mathrm{SAS}+0 \mathrm{~kg} \mathrm{~N} / \mathrm{ha}+60 \mathrm{~kg} \mathrm{P} \mathrm{O}_{5} / \mathrm{ha}+0 \mathrm{~T}$ fumier $/ \mathrm{ha}$ \\
\hline $\mathrm{T}_{1} \mathrm{E}_{3}$ & 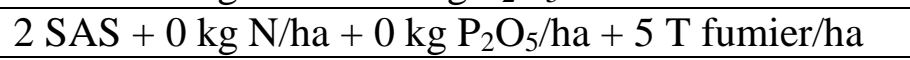 \\
\hline $\mathrm{T}_{1} \mathrm{E}_{4}$ & $2 \mathrm{SAS}+40 \mathrm{~kg} \mathrm{~N} / \mathrm{ha}+60 \mathrm{~kg} \mathrm{P}_{2} \mathrm{O}_{5} / \mathrm{ha}+5 \mathrm{~T}$ fumier/ha \\
\hline $\mathrm{T}_{2} \mathrm{E}_{0}$ & 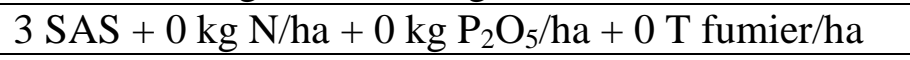 \\
\hline $\mathrm{T}_{2} \mathrm{E}_{1}$ & 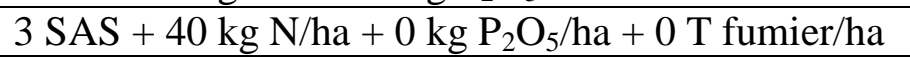 \\
\hline $\mathrm{T}_{2} \mathrm{E}_{2}$ & 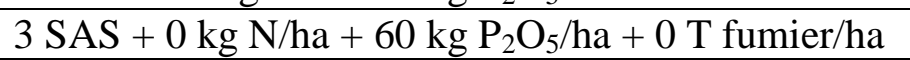 \\
\hline $\mathrm{T}_{2} \mathrm{E}_{3}$ & $3 \mathrm{SAS}+0 \mathrm{~kg} \mathrm{~N} / \mathrm{ha}+0 \mathrm{~kg} \mathrm{P}_{2} \mathrm{O}_{5} / \mathrm{ha}+5 \mathrm{~T}$ fumier/ha \\
\hline $\mathrm{T}_{2} \mathrm{E}_{4}$ & 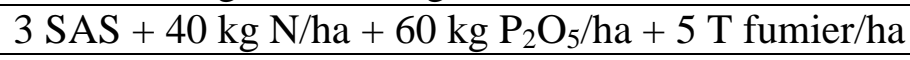 \\
\hline $\mathrm{T}_{3} \mathrm{E}_{0}$ & $4 \mathrm{SAS}+0 \mathrm{~kg} \mathrm{~N} / \mathrm{ha}+0 \mathrm{~kg} \mathrm{P}_{2} \mathrm{O}_{5} / \mathrm{ha}+0 \mathrm{~T}$ fumier/ha \\
\hline $\mathrm{T}_{3} \mathrm{E}_{1}$ & 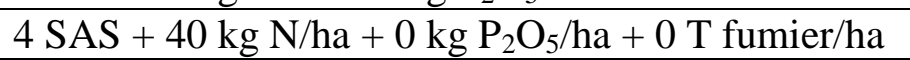 \\
\hline $\mathrm{T}_{3} \mathrm{E}_{2}$ & $4 \mathrm{SAS}+0 \mathrm{~kg} \mathrm{~N} / \mathrm{ha}+60 \mathrm{~kg} \mathrm{P} \mathrm{O}_{5} / \mathrm{ha}+0 \mathrm{~T}$ fumier/ha \\
\hline $\mathrm{T}_{3} \mathrm{E}_{3}$ & $4 \mathrm{SAS}+0 \mathrm{~kg} \mathrm{~N} / \mathrm{ha}+0 \mathrm{~kg} \mathrm{P}_{2} \mathrm{O}_{5} / \mathrm{ha}+5 \mathrm{~T}$ fumier/ha \\
\hline $\mathrm{T}_{3} \mathrm{E}_{4}$ & $4 \mathrm{SAS}+40 \mathrm{~kg} \mathrm{~N} / \mathrm{ha}+60 \mathrm{~kg} \mathrm{P}_{2} \mathrm{O}_{5} / \mathrm{ha}+5 \mathrm{~T}$ fumier $/ \mathrm{ha}$ \\
\hline
\end{tabular}

\subsection{Pratiques agronomiques et collectes des données}

Cinq plantes choisies au hasard ont été marqués et étiquetés de quatre lignes du milieu de chaque traitement pour diverses observations et les données recueillies ont été analysées statistiquement tel que décrite en suivant la procédure Gomez et Gomez (1984). La hauteur de la plante a été mesurée de la base de la plante au bourgeon apical de la plante et exprimée en centimètres. La surface totale de la feuille a été enregistrée par la mesure de longueur maximale (L) et la largeur (l) de feuilles trifoliées et en multipliant (L x l) par un facteur de correction de 0,6. La biomasse aérienne sèche a été obtenu par cinq plantes choisies au hasard, puis coupé au niveau du collet et divisé en différentes parties à savoir, les feuilles, la tige et parties reproductrices. Les différentes parties d'échantillons ont été séchés à l'étuve à $80^{\circ} \mathrm{C}$ jusqu'à poids constant et les poids ont été enregistrés. Les moyennes de biomasse aérienne ont été ramené en $\left(\mathrm{kg} \mathrm{ha}^{-1}\right)$. Pour les paramètres des nodules, le nombre, l'efficacité et le poids sec des nodules ont été évalués. Le nombre des nodules par plante a été obtenu par comptage manuel des nodules présentes sur les racines de la plante de haricot après son déracinement à 4 et 6 SAS. Effectivité des nodules a été obtenue à 4 et 6 SAS après comptage manuel des nodules. Le principe consiste à sélectionner 15 nodules au hasard et en faisant une 
coupe longitudinale de chacune d'eux à l'aide d'un rasoir ; les nodules présentant la coloration rougeâtre ou son équivalent (rosée, orangée, etc.) ont été considéré effectives c'est-à-dire fixatrice d'azote et dans l'autre cas, si cette coloration est blanchâtre ou verdâtre, ces nodules sont dits non effectives (non fixatrices d'azote). L'effectivité des nodules était présentée en pourcentage. Poids sec des nodules a été obtenu après le comptage et le calcul de l'effectivité des nodules. Tous les nodules obtenus par plante sont mis dans une enveloppe sac pour un dessèchement à l'étuve à une température de $70^{\circ} \mathrm{C}$ durant $72 \mathrm{~h}$. Le poids sec des nodules a été obtenu à l'aide d'une balance de précision et il est exprimé en gramme. Le poids de 100 graines a été obtenue par peser sur une balance de précision de 100 graines pris au hasard dans parmi les graines obtenues sur la parcelle utile après épluchage. Le rendement/ha est le poids de l'ensemble des graines de chaque parcelle rapportée à l'hectare par la méthode de règle de trois simples. L'indice de récolte a été obtenu par un rapport entre le rendement en graine $\left(\mathrm{kg} \mathrm{ha}^{-1}\right)$ et la biomasse totale obtenue à la récolte (rendement en graine + biomasse aérienne) $\left(\mathrm{kg} \mathrm{ha}^{-1}\right)$. En ce qui concerne l'analyse économique, la technique de budget, décrit dans Noronha (1987) a été utilisé, et aussi dans l'analyse des expériences menées par Teixeira Filho et al. (2010), Binotti et al. (2010) et Sabundjian, et al. (2014). La meilleure alternative est celle offrant des avantages nets ou plus les marges bénéficiaires (Teixeira Filho et al., 2010). Les résultats ont été calculés à partir du prix des entrées et des sorties de l'expérimentation et les couts de l'engrais. Le rapport bénéfice-coût (BC) a été calculé en utilisant la formule suivante :

$$
\text { Revenu Brut (ha } \left.{ }^{-1}\right)
$$

$\mathrm{BC}=\frac{\text { Coût du traitement engrais }\left(\mathrm{ha}^{-1}\right)}{\mathrm{R}}$

\subsection{Analyse statistique}

Avant les analyses, les données ont été encodées à l'aide du tableur Microsoft Excel 2016 et pour effectuer les graphiques présentés en fonction de différents traitements. Le calcul des fréquences et de l'abondance moyenne a été réalisé avec le même tableur. Le paramètre végétatif ainsi que les composantes du rendement et le rendement ont soumis au test d'analyse de la variance (ANOVA), au seuil de signification de 5\%, à l'aide du logiciel Genstat version 15. Le test de LSD a été utilisé pour séparer les moyennes.

\section{Résultats}

\subsection{Hauteurs des plants}

La figure 1 présente les différentes hauteurs des plants de haricot à 3, 4, 5 et 6 SAS haricot affectés par les différentes dates d'application (2, 3 et 4 SAS) d'application d'engrais.
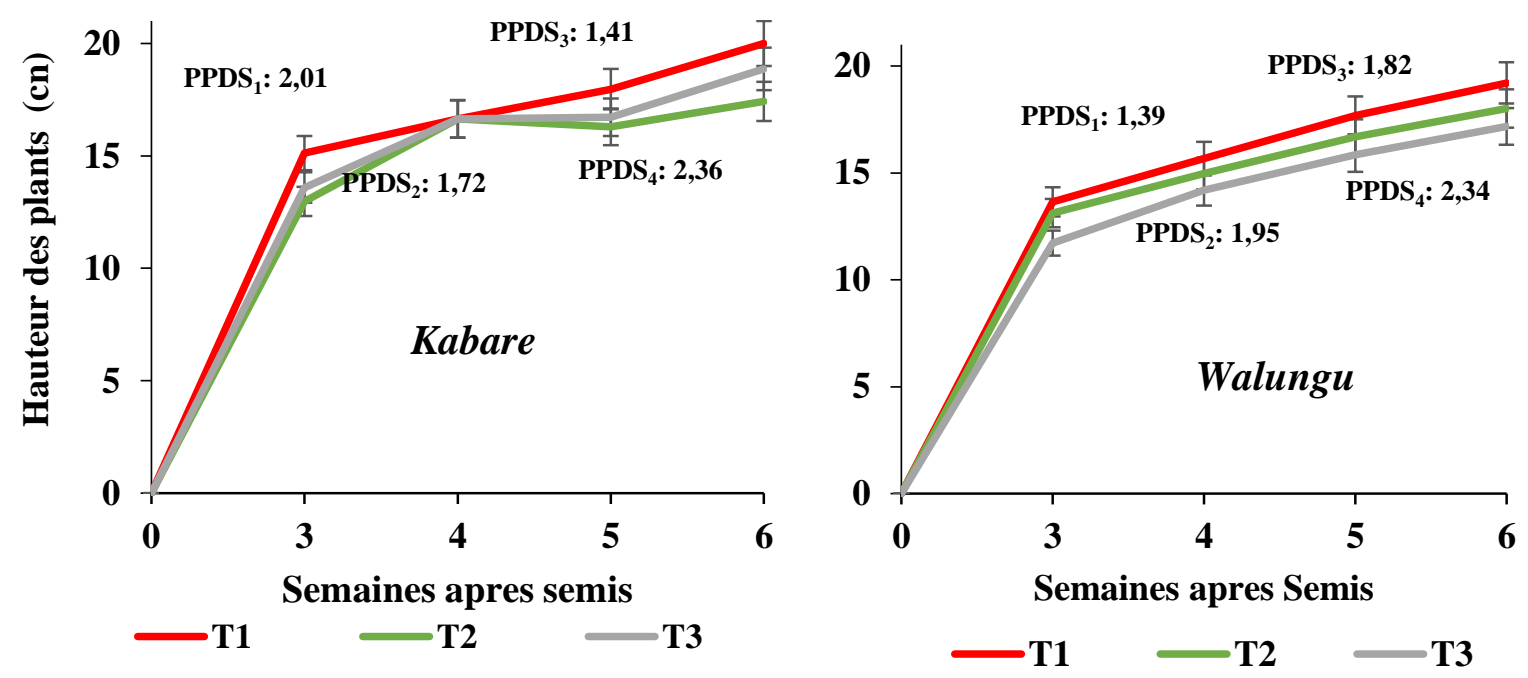

Temps d'application de l'engrais organo-minéral $\left(\mathbf{T}_{1}, \mathbf{T}_{2}\right.$ et $\left.\mathbf{T}_{3}\right)$

Figure 1 : Hauteurs des plants de haricot affectés par les différentes dates d'application (2, 3 et 4 SAS) d'application d'engrais.

La figure 2 présente les différentes hauteurs des plants de haricot à 3, 4, 5 et 6 SAS affectée par les différentes options de GIFS. 

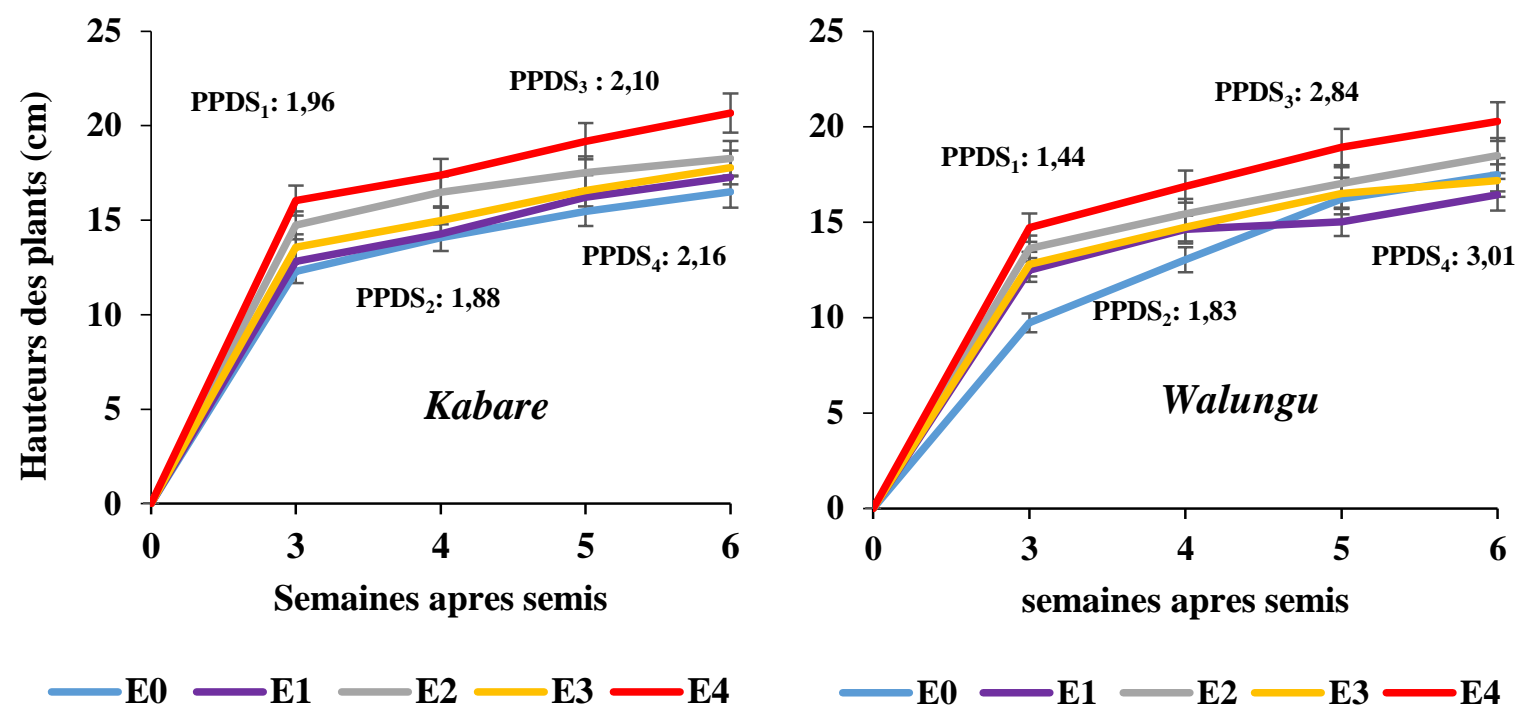

Différentes options de la gestion intégrée de la fertilité de sol $\left(E_{0}, E_{1}, E_{2}, E_{3}\right.$ et $\left.E_{4}\right)$

Figure 2 : Hauteurs des plants de haricot affectés par les différentes options de la gestion intégrée de la fertilité du sol (GIFS).

Il ressort des résultats (Figures 1 et 2) que les différentes hauteurs collectées à différents temps de collecte des données (3, 4, 5 et $6 \mathrm{SAS})$ ont été influencé par les différentes périodes d'application d'engrais et les options GIFS utilisées au seuil de probabilité de 5\% dans les différents sites d'études (Kabare et Walungu). Ces mêmes résultats montrent que la meilleure date d'application d'engrais est 2 semaines après semis (2 SAS) et la meilleure option GIFS est la combinaison de $40 \mathrm{~kg} \mathrm{~N} \mathrm{ha}^{-1}+60 \mathrm{~kg} \mathrm{P}_{2} \mathrm{O}_{5} \mathrm{ha}^{-1}+5 \mathrm{~T}_{\text {fumier ha }}{ }^{-1}$. Au vu des résultats des figures 1 et 2 , les plants de Kabare ont donnée des hauteurs supérieures par rapport à celles de Walungu.

\subsection{Surface foliaire des plants}

La figure 3 présente les différentes surfaces foliaires des plants de haricot à 3, 4, 5 et 6 SAS haricot affectés par les différentes dates d'application (2, 3 et 4 SAS) d'application d'engrais.

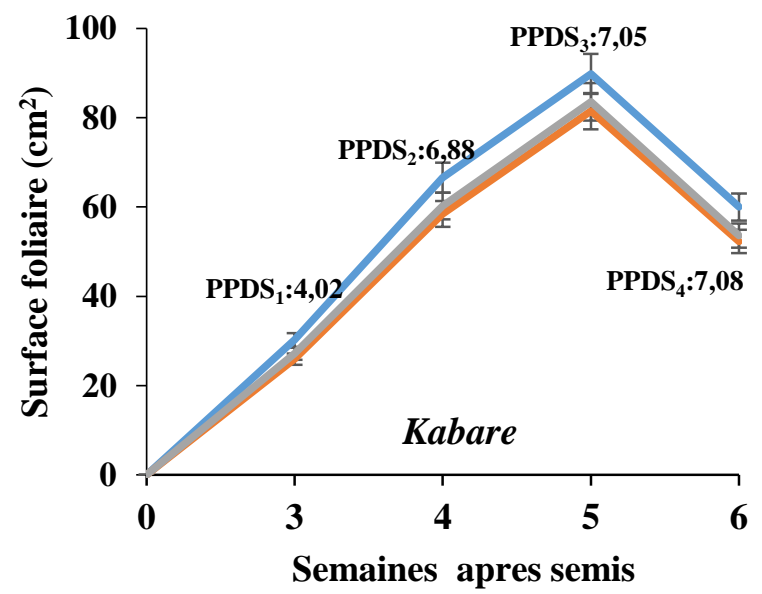

2 SAS $\longrightarrow$ SAS $\longrightarrow$ SAS

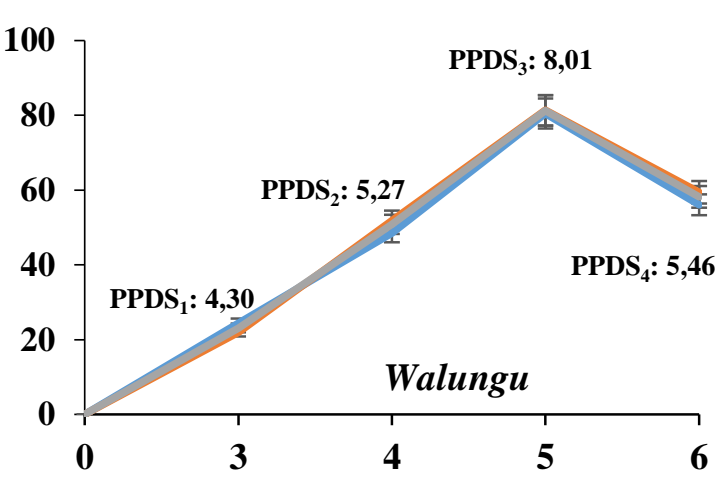

Semaines apres semis

2 SAS -3 SAS $\longrightarrow$ SAS

Temps d'application de l'engrais organo-minéral ( $\mathbf{T}_{1}, \mathbf{T}_{\mathbf{2}}$ et $\left.\mathbf{T}_{\mathbf{3}}\right)$

Figure 3 : Surfaces foliaires des plants de haricot affectés par les différentes dates d'application (2, 3 et 4 SAS) d'application d'engrais.

La figure 4 présente les différentes surfaces foliaires des plants de haricot à 3, 4, 5 et 6 SAS affectée par les différentes options de GIFS. 

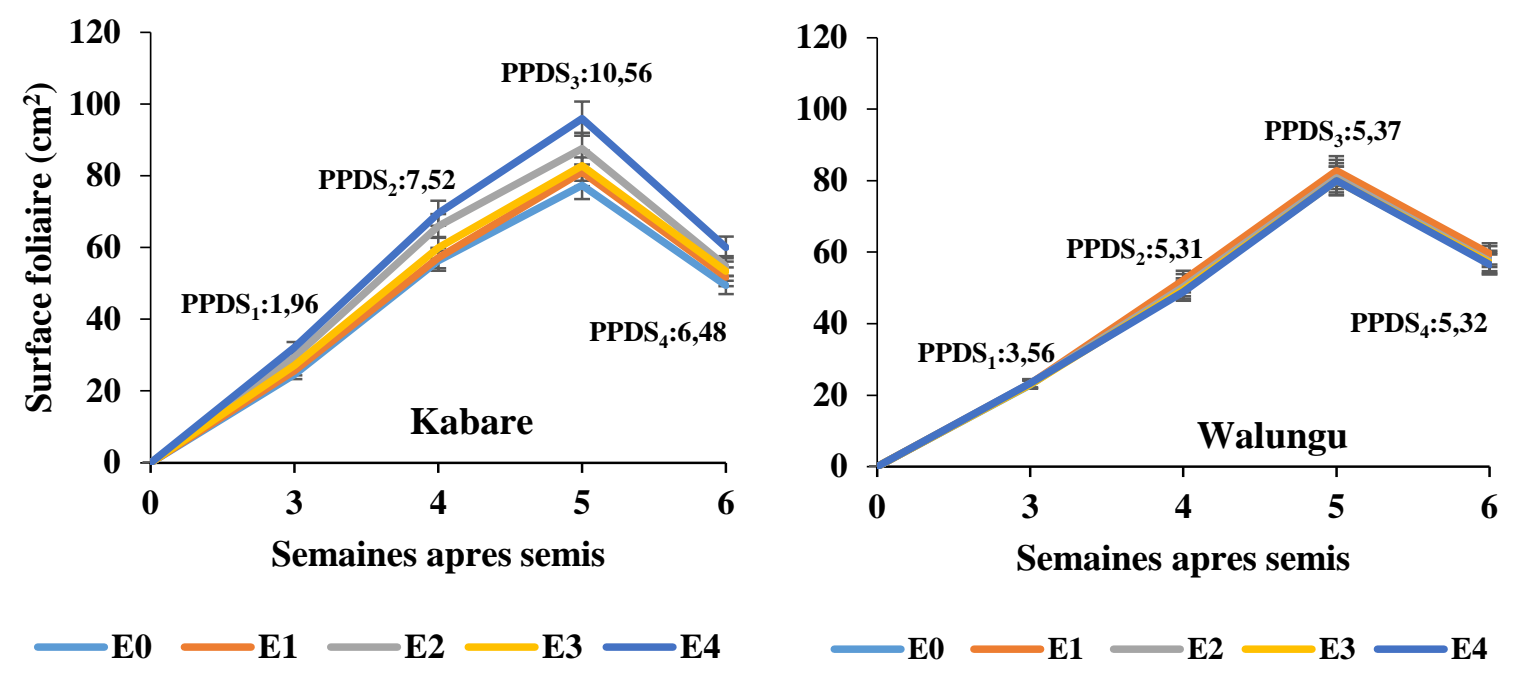

Différentes options de la gestion intégrée de la fertilité de sol $\left(\mathbf{E}_{\mathbf{0}}, \mathbf{E}_{\mathbf{1}}, \mathbf{E}_{\mathbf{2}}, \mathbf{E}_{\mathbf{3}}\right.$ et $\left.\mathbf{E}_{\mathbf{4}}\right)$

Figure 4 : Surfaces foliaires des plants de haricot affectés par les différentes options de la gestion intégrée de la fertilité du sol (GIFS).

En se référant aux figures 3 et 4 ci-haut, nous constatons que la valeur de la surface foliaire augmente avec le temps jusqu'à la $5^{\text {ème }}$ Semaines après semis et puis il s'en suit une décroissance dans les deux sites d'études (Kabare et Walungu). Les différentes surfaces foliaires collectées à Kabare suivent la même allure que les différentes valeurs des hauteurs des plants (Figure 1 et 2). Alors que la différence a été significative $(\mathrm{p}<0,05)$ pour les plants de Kabare quant aux différentes options utilisées et le temps d'application de l'engrais contrairement aux plants de Walungu qui n'ont pas présenté de différence ( $p>0,05)$ au même seuil.

\subsection{Biomasse aérienne}

La figure 5 présente les différentes biomasses aériennes des plants de haricot à 3, 4, 5 et 6 SAS haricot affectés par les différentes dates d'application (2, 3 et 4 SAS) d'application d'engrais.
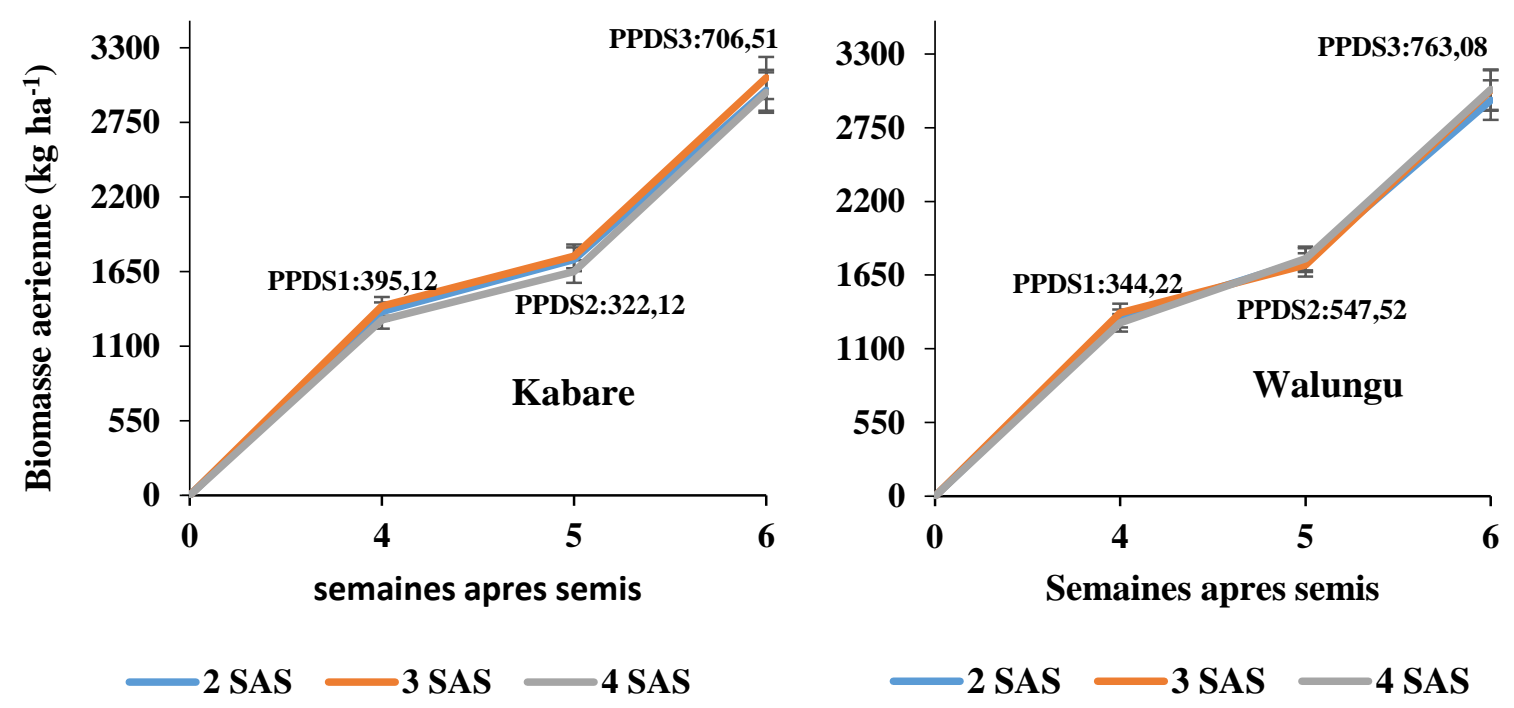

Temps d'application de l'engrais organo-minéral $\left(\mathbf{T}_{\mathbf{1}}, \mathbf{T}_{\mathbf{2}}\right.$ et $\left.\mathbf{T}_{\mathbf{3}}\right)$

Figure 5 : Biomasses aériennes des plants de haricot affectés par les différentes dates d'application (2, 3 et 4 SAS) d'application d'engrais.

La figure 6 présente les différentes biomasses aériennes des plants de haricot à 3, 4, 5 et 6 SAS affectée par les différentes options de GIFS. 


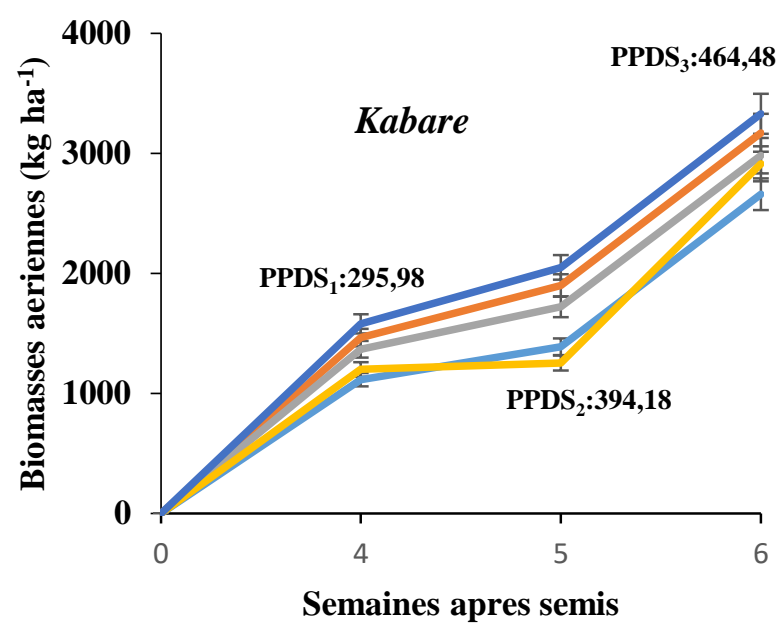

$\longrightarrow \mathrm{E} 0-\mathrm{E} 1-\mathrm{E} 2-\mathrm{E} 3-\mathrm{E} 4$

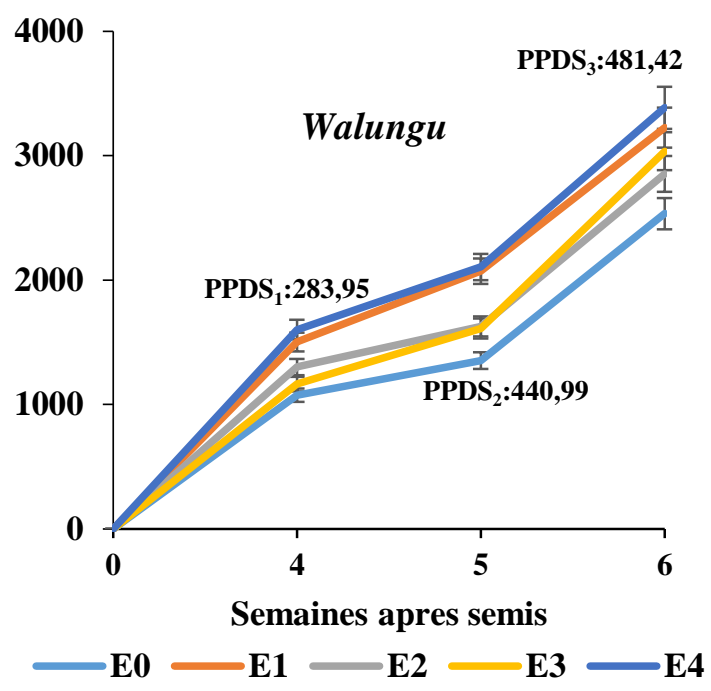

Différentes options de la gestion intégrée de la fertilité de sol $\left(E_{0}, E_{1}, E_{2}, E_{3}\right.$ et $\left.E_{4}\right)$

Figure 6 : Biomasses aériennes des plants de haricot affectés par les différentes options de la gestion intégrée de la fertilité du sol (GIFS).

Les résultats (Figures 5 et 6) montrent que seules les différentes périodes d'applications n'ont pas influencées pas les biomasses aériennes. Mais si ces dates sont combinées aux différentes options d'intensification, il y a différence $(\mathrm{p}<0,05)$. Ces résultats ont montré que 2 SAS s'avère la meilleure date d'application d'engrais à toutes les périodes de collectes des données et la meilleure combinaison de

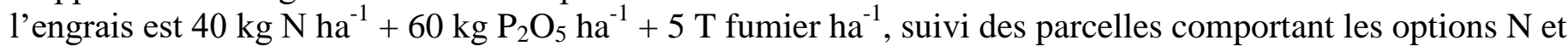
$\mathrm{P}$, puis celles de fumier et enfin le témoin. Cette allure est valable dans les deux sites expérimentaux d'étude (Kabare et Walungu).

\subsection{Paramètres de nodulation}

Le tableau 3 présente le nombre, effectivité et le poids de nodules des plants de haricot

Tableau 3 : Nombre, effectivité et le poids de nodules plants de haricot affectés par la fertilisation organominérale à différentes dates d'application (2, 3 et 4 SAS).

\begin{tabular}{|c|c|c|c|c|c|c|c|}
\hline \multicolumn{2}{|c|}{ Temps d'application } & \multicolumn{2}{|c|}{ Nombre des nodules } & \multicolumn{2}{|c|}{ Effectivité des nodules } & \multicolumn{2}{|c|}{ Poids des nodules } \\
\hline & & Kabare & Walungu & Kabare & Walungu & Kabare & Walungu \\
\hline 2 SAS & & $13,13^{\mathrm{ns}}$ & $6,93^{\mathrm{ns}}$ & $90,51^{\mathrm{ns}}$ & $81,31^{\mathrm{ns}}$ & $2,03^{\mathrm{ns}}$ & $1,41^{\mathrm{ns}}$ \\
\hline $3 \mathrm{SAS}$ & & $11,97^{\mathrm{ns}}$ & $8,67_{\mathrm{ns}}$ & $83,38^{\mathrm{ns}}$ & $76,78^{\mathrm{ns}}$ & $1,90^{\mathrm{ns}}$ & $1,54^{\mathrm{ns}}$ \\
\hline $4 \mathrm{SAS}$ & & $11,60^{\mathrm{ns}}$ & $8,00^{\text {ns }}$ & $86,71^{\mathrm{ns}}$ & $76,11^{\mathrm{ns}}$ & $1,77^{\mathrm{ns}}$ & $1,47^{\mathrm{ns}}$ \\
\hline PPDS & & 4,76 & 3,87 & 10,15 & 7,15 & 0,23 & 0,13 \\
\hline \multicolumn{8}{|c|}{ Fertilisants } \\
\hline \multicolumn{2}{|c|}{$\mathrm{E}_{0}$} & $8,61^{b}$ & $7,44^{b}$ & $82,48^{b}$ & $75,15^{b}$ & $1,66^{\mathrm{b}}$ & $1,26^{\mathrm{b}}$ \\
\hline \multicolumn{2}{|l|}{ E1 } & $10,44^{b}$ & $8,11^{\mathrm{a}}$ & $82,11^{b}$ & $77,11^{\mathrm{a}}$ & $1,82^{b}$ & $1,33^{b}$ \\
\hline \multicolumn{2}{|l|}{ E2 } & $13,78^{\mathrm{a}}$ & $6,11^{\mathrm{a}}$ & $90,85^{\mathrm{a}}$ & $80,85^{\mathrm{a}}$ & $2,33^{\mathrm{a}}$ & $1,77^{\mathrm{a}}$ \\
\hline \multicolumn{2}{|l|}{ E3 } & $13,00^{\mathrm{a}}$ & $8,67^{\mathrm{a}}$ & $89,37^{\mathrm{a}}$ & $77,37^{\mathrm{a}}$ & $1,76^{b}$ & $1,46^{\mathrm{a}}$ \\
\hline \multicolumn{2}{|l|}{ E4 } & $15,33^{\mathrm{a}}$ & $9,00^{\mathrm{a}}$ & $89,52^{\mathrm{a}}$ & $77,85^{\mathrm{a}}$ & $1,93^{\mathrm{a}}$ & $1,56^{\mathrm{a}}$ \\
\hline \multicolumn{2}{|c|}{ PPDS } & 4,61 & 1,44 & 6,48 & 3,15 & 0,43 & 0,39 \\
\hline \multicolumn{8}{|c|}{ Interaction } \\
\hline \multirow[t]{4}{*}{2 SAS } & $\mathrm{E}_{0}$ & $9,00^{\mathrm{ns}}$ & $5,00^{\text {ns }}$ & $80,89^{b}$ & $74,89^{b}$ & $1,69^{b}$ & $1,09^{b}$ \\
\hline & E1 & $11,00^{\mathrm{ns}}$ & $7,00^{\mathrm{ns}}$ & $88,11^{\mathrm{a}}$ & $78,11^{b}$ & $1,84^{\mathrm{ab}}$ & $1,34^{\mathrm{ab}}$ \\
\hline & E2 & $16,00^{\mathrm{ns}}$ & $6,00^{\mathrm{ns}}$ & $97,89^{\mathrm{a}}$ & $87,89^{\mathrm{a}}$ & $2,83^{\mathrm{a}}$ & $1,73^{\mathrm{ab}}$ \\
\hline & E3 & $12,00^{\mathrm{ns}}$ & $7,00^{\mathrm{ns}}$ & $90,33^{\mathrm{a}}$ & $75,33^{b}$ & $1,82^{b}$ & $1,62^{\mathrm{ab}}$ \\
\hline
\end{tabular}




\begin{tabular}{|c|c|c|c|c|c|c|c|}
\hline & E4 & $17,67^{\mathrm{ns}}$ & $9,67^{\mathrm{ns}}$ & $95,33^{\mathrm{a}}$ & $90,33^{\mathrm{a}}$ & $1,98^{\mathrm{ab}}$ & $1,28^{\mathrm{ab}}$ \\
\hline \multirow[t]{5}{*}{$3 \mathrm{SAS}$} & $\mathrm{E}_{0}$ & $8,50^{\mathrm{ns}}$ & $8,00^{\mathrm{ns}}$ & $78,78^{\mathrm{a}}$ & $74,78^{b}$ & $1,73^{b}$ & $1,43^{\mathrm{ab}}$ \\
\hline & E1 & $11,00^{\mathrm{nS}}$ & $9,00^{\text {ns }}$ & $86,22^{\mathrm{ab}}$ & $76,22^{b}$ & $1,91^{\mathrm{ab}}$ & $1,42^{\mathrm{ab}}$ \\
\hline & E2 & $12,67^{\mathrm{ns}}$ & $6,67^{\text {ns }}$ & $88,67^{\mathrm{a}}$ & $78,67^{b}$ & $2,21^{\mathrm{ab}}$ & $1,71^{\mathrm{ab}}$ \\
\hline & E3 & $13,67^{\mathrm{ns}}$ & $10,67^{\mathrm{ns}}$ & $89,33^{\mathrm{a}}$ & $79,33^{b}$ & $1,77^{b}$ & $1,47^{\mathrm{ab}}$ \\
\hline & E4 & $14,00^{\mathrm{ns}}$ & $9,00^{\mathrm{ns}}$ & $73,89^{b}$ & $81,89^{\mathrm{ab}}$ & $1,87^{\mathrm{ab}}$ & $1,67^{\mathrm{ab}}$ \\
\hline \multirow[t]{5}{*}{$4 \mathrm{SAS}$} & $\mathrm{E}_{0}$ & $8,33^{\text {ns }}$ & $9,33^{\text {ns }}$ & $87,78^{\mathrm{a}}$ & $74,78^{b}$ & $1,55^{\mathrm{b}}$ & $1,25^{\mathrm{ab}}$ \\
\hline & E1 & $9,33^{\mathrm{ns}}$ & $8,33^{\text {ns }}$ & $93,00^{\mathrm{a}}$ & $77,00^{b}$ & $1,72^{b}$ & $1,22^{\mathrm{ab}}$ \\
\hline & E2 & $12,67^{\mathrm{ns}}$ & $5,67^{\text {ns }}$ & $86,00^{\mathrm{ab}}$ & $76,00^{b}$ & $1,96^{\mathrm{ab}}$ & $1,86^{\mathrm{a}}$ \\
\hline & E3 & $13,33^{\mathrm{ns}}$ & $8,33^{\text {ns }}$ & $88,45^{\mathrm{a}}$ & $77,45^{\mathrm{b}}$ & $1,70^{b}$ & $1,30^{\mathrm{ab}}$ \\
\hline & E4 & $14,33^{\mathrm{ns}}$ & $8,33^{\mathrm{ns}}$ & $78,33^{b}$ & $78,33^{\mathrm{b}}$ & $1,94^{\mathrm{ab}}$ & $1,74^{\mathrm{ab}}$ \\
\hline \multicolumn{2}{|c|}{ PPDS } & 10,23 & 6,13 & 12,40 & 10,88 & 1,01 & 0,72 \\
\hline \multicolumn{2}{|c|}{$\mathrm{CV}(\%)$} & 4,70 & 6,30 & 8,51 & 5,65 & 6,65 & 7,65 \\
\hline
\end{tabular}

Il ressort de ce tableau 3 que les paramètres de nodulation (nombre, effectivité et le poids sec des nodules) collectées à des différentes dates n'ont pas présentées de différence significative au seuil de probabilité de 5 $\%$ pour les dates d'application d'engrais (2, 3 et $4 \mathrm{SAS})$ mais seulement pour types d'engrais organominéraux utilisés. La meilleure combinaison de l'engrais est $40 \mathrm{~kg} \mathrm{~N} \mathrm{ha}^{-1}+60 \mathrm{~kg} \mathrm{P}_{2} \mathrm{O}_{5} \mathrm{ha}^{-1}+5 \mathrm{~T}_{\text {fumier ha }}$ ${ }^{1}$ à toutes les périodes de collectes des données $(3,4,5$ et $6 \mathrm{SAS})$. L'interaction des traitements a présenté des différences significatives $(\mathrm{p}<0,05)$ excepté pour le nombre des nodules et la combinaison 2 SAS et 40 $\mathrm{kg} \mathrm{N} \mathrm{ha}^{-1}+60 \mathrm{~kg} \mathrm{P}_{2} \mathrm{O}_{5} \mathrm{ha}^{-1}+5 \mathrm{~T}_{\text {fumier ha }}{ }^{-1}$ s'est avérée meilleure.

\subsection{Paramètres de rendement}

Le tableau 4 présente le poids de 100 graines, rendement par hectare et l'indice de récolte.

Tableau 4 : Poids de 100 graines, rendement par hectare et indice de récolte affectés par la fertilisation organon- minérale à différentes dates d'application (2, 3 et 4 SAS).

\begin{tabular}{|c|c|c|c|c|c|c|c|}
\hline \multicolumn{2}{|c|}{$\begin{array}{l}\text { Temps } \\
\text { d'application }\end{array}$} & \multicolumn{2}{|c|}{ Poids de 100 graines } & \multicolumn{2}{|c|}{ Indice de récolte } & \multicolumn{2}{|c|}{ Rendement/ha } \\
\hline & & Kabare & Walungu & Kabare & Walungu & Kabare & Walungu \\
\hline 2 SAS & & $35,62^{\mathrm{ns}}$ & $31,76^{\mathrm{ns}}$ & $50,78^{\mathrm{ns}}$ & $46,30^{\mathrm{ns}}$ & $1483,78^{\mathrm{ns}}$ & $1342,50^{\mathrm{ns}}$ \\
\hline $3 \mathrm{SAS}$ & & $34,52^{\mathrm{ns}}$ & $35,80^{\text {ns }}$ & $46,54^{\mathrm{ns}}$ & $45,00^{\mathrm{ns}}$ & $1414,43^{\mathrm{ns}}$ & $1361,00^{\mathrm{ns}}$ \\
\hline $4 \mathrm{SAS}$ & & $35,38^{\mathrm{ns}}$ & $37,08^{\text {ns }}$ & $45,64^{\mathrm{ns}}$ & $45,00^{\mathrm{ns}}$ & $1362,21^{\mathrm{ns}}$ & $1320,83^{\mathrm{ns}}$ \\
\hline PPDS & & 17,54 & 18,11 & 5,37 & 19,20 & 209,9 & 263,71 \\
\hline \multicolumn{8}{|c|}{ Fertilisants } \\
\hline \multicolumn{2}{|c|}{$\mathrm{E}_{0}$} & $32,99^{b}$ & $31,61^{b}$ & $41,68^{b}$ & $34,70^{b}$ & $1056,42^{d}$ & $866,67^{\mathrm{d}}$ \\
\hline \multicolumn{2}{|l|}{ E1 } & $36,34^{\mathrm{ab}}$ & $34,93^{\mathrm{ab}}$ & $42,61^{b}$ & $37,70^{b}$ & $1328,11^{\mathrm{c}}$ & $1166,67^{\mathrm{c}}$ \\
\hline \multicolumn{2}{|l|}{ E2 } & $34,84^{\mathrm{ab}}$ & $35,62^{\mathrm{ab}}$ & $52,16^{\mathrm{a}}$ & $54,40^{\mathrm{a}}$ & $1494,47^{b}$ & $1537,78^{b}$ \\
\hline \multicolumn{2}{|l|}{ E3 } & $34,02^{b}$ & $35,37^{\mathrm{ab}}$ & $48,36^{\mathrm{a}}$ & $49,30^{\mathrm{a}}$ & $1440,02^{b}$ & $1434,72^{b}$ \\
\hline \multicolumn{2}{|l|}{ E4 } & $37,67^{\mathrm{a}}$ & $36,88^{\mathrm{a}}$ & $53,24^{\mathrm{a}}$ & $51,10^{\mathrm{a}}$ & $1781,23^{\mathrm{a}}$ & $1701,39^{\mathrm{a}}$ \\
\hline \multicolumn{2}{|c|}{ PPDS } & 3,48 & 4,41 & 5,36 & 6,80 & 155,01 & 155,72 \\
\hline \multicolumn{8}{|c|}{ Interaction } \\
\hline \multirow[t]{5}{*}{2 SAS } & $\mathrm{E}_{0}$ & $28,40^{b}$ & $29,33^{b}$ & $45,55^{\mathrm{b}}$ & $37,80^{b}$ & $1073,14^{\mathrm{c}}$ & $879,17^{\mathrm{c}}$ \\
\hline & E1 & $36,01^{\mathrm{a}}$ & $28,33^{b}$ & $46,80^{b}$ & $44,60^{\mathrm{ab}}$ & $1350,03^{b}$ & $1279,17^{b}$ \\
\hline & E2 & $38,39^{\mathrm{a}}$ & $31,16^{\mathrm{ab}}$ & $55,18^{\mathrm{a}}$ & $51,70^{\mathrm{a}}$ & $1640,55^{\mathrm{a}}$ & $1512,50^{\mathrm{a}}$ \\
\hline & E3 & $36,07^{\mathrm{a}}$ & $32,49^{\mathrm{ab}}$ & $50,00^{\mathrm{a}}$ & $41,10^{b}$ & $1550,23^{\mathrm{a}}$ & $1400,00^{\mathrm{a}}$ \\
\hline & E4 & $39,06^{\mathrm{a}}$ & $44,51^{\mathrm{a}}$ & $56,35^{\mathrm{a}}$ & $51,50^{\mathrm{a}}$ & $1804,93^{\mathrm{a}}$ & $1641,67^{\mathrm{a}}$ \\
\hline \multirow[t]{5}{*}{$3 \mathrm{SAS}$} & $\overline{E_{0}}$ & $27,29^{b}$ & $27,29^{b}$ & $39,81^{b}$ & $33,20^{b}$ & $1052,22^{\mathrm{c}}$ & $870,83^{c}$ \\
\hline & E1 & $40,02^{\mathrm{a}}$ & $36,22^{a b}$ & $41,62^{b}$ & $36,30^{b}$ & $1306,24^{\mathrm{bc}}$ & $1145,83^{b}$ \\
\hline & E2 & $36,39^{\mathrm{a}}$ & $37,46^{\mathrm{ab}}$ & $51,29^{\mathrm{a}}$ & $52,00^{\mathrm{a}}$ & $1487,36^{b}$ & $1488,33^{\mathrm{a}}$ \\
\hline & E3 & $29,88^{b}$ & $43,06^{\mathrm{a}}$ & $50,02^{\mathrm{a}}$ & $52,60^{\mathrm{a}}$ & $1456,32^{b}$ & $1512,50^{\mathrm{a}}$ \\
\hline & E4 & $40,23^{\mathrm{a}}$ & $31,92^{\mathrm{ab}}$ & $50,03^{\mathrm{a}}$ & $51,10^{\mathrm{a}}$ & $1769,36^{\mathrm{a}}$ & $1787,50^{\mathrm{a}}$ \\
\hline
\end{tabular}




\begin{tabular}{|l|l|c|c|c|c|c|c|}
\hline 4 SAS & $\mathrm{E}_{0}$ & $31,92 \mathrm{~b}$ & $37,00^{\mathrm{ab}}$ & $39,68^{\mathrm{b}}$ & $33,10^{\mathrm{b}}$ & $1042,33^{\mathrm{c}}$ & $850,00^{\mathrm{c}}$ \\
\hline & $\mathrm{E} 1$ & $37,03^{\mathrm{a}}$ & $40,23^{\mathrm{ab}}$ & $39,40^{\mathrm{b}}$ & $32,10^{\mathrm{b}}$ & $1328,10^{\mathrm{bc}}$ & $1075,00^{\mathrm{b}}$ \\
\hline & $\mathrm{E} 2$ & $37,29^{\mathrm{a}}$ & $36,39^{\mathrm{ab}}$ & $50,01^{\mathrm{a}}$ & $59,50^{\mathrm{a}}$ & $1356,23^{\mathrm{b}}$ & $1612,50^{\mathrm{a}}$ \\
\hline & $\mathrm{E} 3$ & $33,38^{\mathrm{a}}$ & $35,09^{\mathrm{ab}}$ & $45,07^{\mathrm{b}}$ & $49,30^{\mathrm{a}}$ & $1312,24^{\mathrm{bc}}$ & $1391,67^{\mathrm{a}}$ \\
\hline & $\mathrm{E} 4$ & $41,44^{\mathrm{a}}$ & $36,72^{\mathrm{ab}}$ & $53,36^{\mathrm{a}}$ & $50,80^{\mathrm{a}}$ & $1771,11^{\mathrm{a}}$ & $1675,00^{\mathrm{a}}$ \\
\hline CV (\%) & $\mathbf{6 , 3 0}$ & $\mathbf{7 , 4 0}$ & $\mathbf{5 , 8 0}$ & $\mathbf{9 , 7 1}$ & $\mathbf{5 , 6 0}$ & $\mathbf{5 , 9 5}$ \\
\hline PPDS & $\mathbf{9 , 2 5}$ & $\mathbf{1 3 , 6 0}$ & $\mathbf{9 , 1 5}$ & $\mathbf{1 5 , 4}$ & $\mathbf{2 8 6 , 8 1}$ & $\mathbf{3 1 6 , 0 7}$ \\
\hline
\end{tabular}

Le tableau 4 montre que le poids de 100 graines n'a pas présenté de différence significative $(\mathrm{p}<0,05)$ pour les dates d'application d'engrais mais seulement pour les types d'engrais organo-minéraux utilisés. Ce même tableau montre que le rendement et l'indice de récolte ont présentées de différence seulement pour les types d'engrais organo-minéraux utilisés. La meilleure combinaison de l'engrais est $40 \mathrm{~kg} \mathrm{~N} \mathrm{ha}{ }^{-1}+60 \mathrm{~kg}$ $\mathrm{P}_{2} \mathrm{O}_{5} \mathrm{ha}^{-1}+5 \mathrm{~T}$ fumier ha ${ }^{-1}$. L'interaction des traitements a présenté des différences significatives $(\mathrm{p}<0,05)$ pour tous les paramètres de rendement collectés (poids de 100 graines, rendement en graines et l'indice de récolte). La meilleure option de fertilisation est l'application de l'engrais $40 \mathrm{~kg} \mathrm{~N} \mathrm{ha}^{-1}+60 \mathrm{~kg} \mathrm{P}_{2} \mathrm{O}_{5} \mathrm{ha}^{-1}+5$ $\mathrm{T}$ fumier ha ${ }^{-1}$ à 2 semaines après semis.

\subsection{Analyse économique}

La figure 7 présente l'analyse économique affecté par le temps d'application des options de la gestion de la fertilité des sols.

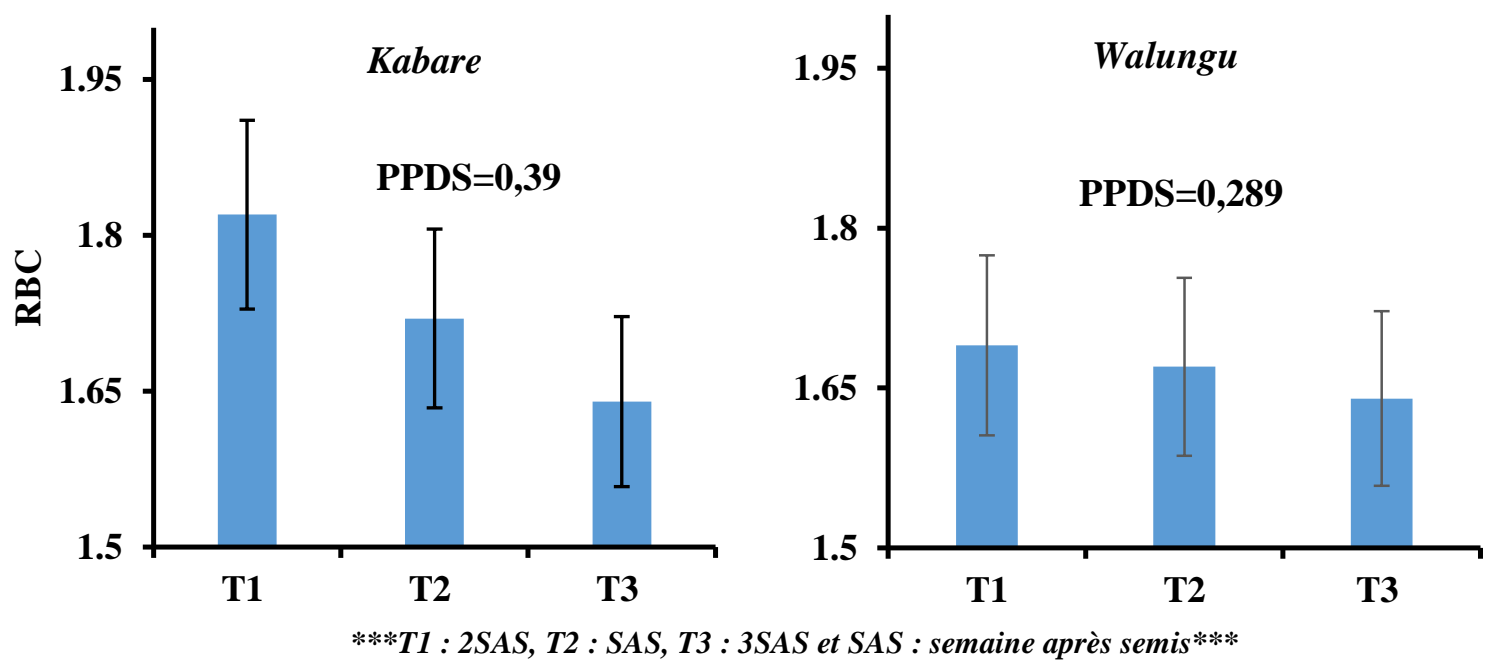

Figure 7 : Analyse économique affectée par les différentes dates d'application d'engrais

La figure 7 montre que seuls les différents temps d'application n'ont pas influencé l'analyse économique (ECOANA) de haricot bio fortifiée affectée par des différentes options de GIFS en étude.

La figure 8 présente l'analyse économique du haricot affecté par les différentes options GIFS (engrais minéraux et engrais organiques). 


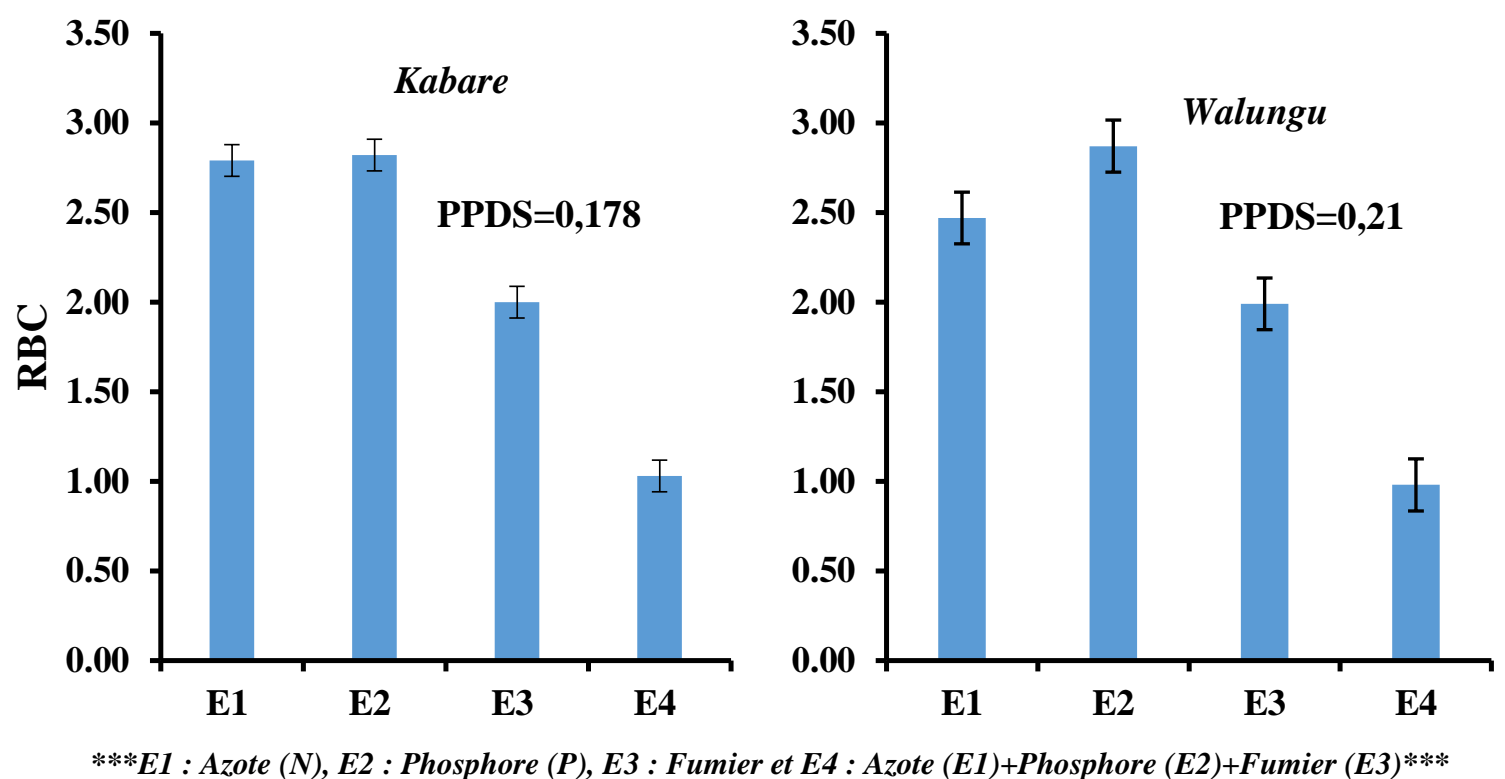

\section{Figure 8 : Analyse économique du haricot affecté par les différentes options de Gestion Intégrée de la Fertilité du Sol (GIFS)}

La figure 8 montre que l'analyse économique a présentée de différence significative au seuil de $5 \%$ pour les différentes options de la gestion intégrée de la fertilité du sol. Les options N (Azote) et P (Phosphore) ont donné la marge bénéficiaire supérieure par rapport autres options avec un RCB de 2,79 pour $\mathrm{N}$ et 2,82 pour $\mathrm{P}$ dans le site de Kabare et le site de Walungu suit la même allure avec un RBC supérieur de 2.

\section{Discussions}

Départ les résultats, nous constatons qu'au Bushi montagneux, il serait préférable de fertiliser avec l'azote et le phosphore avec $40 \mathrm{~kg} \mathrm{~N}^{-1}$ et $60 \mathrm{~kg} \mathrm{P}_{2} \mathrm{O}_{5} \mathrm{ha}^{-1}$. A cela, il faudrait ajouter une fertilisation organique de 5 tonnes par hectare pour une bonne amélioration des conditions des sols. Et également, il a été constaté que l'accroissement de la fertilisation azotée retarde chez le haricot à atteindre la tendreté recherchée à temps. Cette constatation suit la logique qu'un excès d'azote cause un retard de maturité dû à une croissance excessive des organes végétatifs (Eck, 1976 ; Witham et Devlin, 1983). Avec l'augmentation des doses d'azote dans le haricot, les récoltes se feront quelques jours plus tard selon les conditions climatiques d'où la meilleure date d'application est 2 semaines combiné au NP et fumier (Chakirwa, 2011).

Un sommaire de plusieurs recherches effectuées sur le haricot montre qu'il serait avantageux de fractionner les amendements d'azote entre le pré-semis et le début de la croissance (Hochmuth et Hanlon, 2013). La logique de cette pratique vient du fait que l'azote minéral est essentiel à 2 phases du cycle du haricot (Kouassi, 2005). À la levée, car les nodosités ne sont pas encore mises en place. Pendant la maturation des gousses, car même si la fixation fonctionne à son plein potentiel, elle reste insuffisante par rapport aux besoins d'un haricot en plein développement. D'autres études avaient évalué le fractionnement des doses d'azotes à différentes périodes d'application. Cependant, comme la dose appropriée est relativement modeste, le fractionnement pourrait être de $20 \mathrm{~kg} \mathrm{~N} \mathrm{ha}^{-1}$ à chaque application (Chakirwa, 2017).

Les analyses statistiques révèlent que les traitements ont eu d'effets significativement différents $(\mathrm{p}<0,05)$ sur l'indice de récolte. De même, une différence significative $(\mathrm{p}<0,05)$ entre les traitements a été montrée en termes de rendements en graines. Pourtant, les travaux de BADO (2002) prouvent que les apports des engrais combinés aux fumures ont une influence sur les rendements graines. SOME et al. (2014) indiquent que l'application du compost permet d'augmenter le rendement graines, dans cet optique on a pu tester la fertilisation minérale $(\mathrm{N}$ et $\mathrm{P})$ avec le fumier sur la culture de haricot. D'après notre étude, on a pu constater que dans le Bushi montagneux (Kabare et Walungu), le rendement en grains de la culture de haricot dépend de la composition et de la disponibilité des éléments nutritifs du sol. Comme les teneurs en carbone organique et en azote total du sol étaient plus élevées à Kabare, il n'est pas trompeur de dire que la teneur en 
$\mathrm{N}$ et $\mathrm{P}$ disponible est plus élevée ; cela explique en partie le rendement en grains plus élevé observé dans le territoire de Kabare. Ces résultats obtenus comparés aux parcelles témoins n'ont reçu aucun amendement en $\mathrm{N}$ et $\mathrm{P}_{2} \mathrm{O}_{5}$, l'absorption de la culture provenait essentiellement du $\mathrm{N}$ et $\mathrm{P}_{2} \mathrm{O}_{5}$ natif dans le sol. Cela s'est traduit par une absorption plus faible et, par conséquent, les plus petites valeurs de poids de 100 graines et les autres composantes de rendements ont été enregistrées sur ces parcelles.

Ces résultats corroborent avec Kumar et al. (2004), Jagdale et al. (2005) et Veeresh (2003) pour la hauteur des plantes, et Gupta et al. (1996) pour l'indice de surface foliaire et Gupta et al. (1996) and Veeresh (2003) pour production des biomasses sèches formées par plante et Kumar et al. (2004), Thirumalai et Abdul Khalak (1993) et Veeresh (2003) pour le poids de 100 graines, le rendement en graine par hectare et l'indice de récolte du haricot. Il ressort clairement des analyses de sol que toute réponse de la plante à l'application d'engrais NP est susceptible d'être attribuable l'apport des engrais au sol déficient à ces éléments. Visuellement, les plantes dans les parcelles privées de $\mathrm{N}$ et de $\mathrm{P}$ au cours de l'expérimentation dans les deux sites d'études étaient moins saines que les plantes fournies avec $\mathrm{N}$ et $\mathrm{P}$ et cela s'est traduit par des différences significatives de rendement.

Les résultats du tableau 3 montre que dans les parcelles avec un engrais azoté appliqué, la nodulation a été considérablement réduite. Ceci suggère qu'à court terme, avec des cultivars qui se comportent comme Kentwood et Fleetwood, environ $40 \mathrm{~kg} \mathrm{~N} \mathrm{ha}^{-1} \mathrm{du}$ sol peuvent être requis pour un rendement maximum. En pratique, la recommandation pour l'application d'engrais dépend de l'attente de disponibilité de $\mathrm{N}$ par l'apport d'engrais, et de la décomposition des matières organiques. Il semble probable que la recommandation de $20 \mathrm{~kg} \mathrm{~N}^{-1}$ puisse être inadéquate dans certaines circonstances. A en juger par la masse de nodules (Tableau 3) et nombre des nodules par plante (Tableau 3), la fixation de l'azote a commencé à diminuer avant la floraison dans les parcelles fertilisées. L'effectivité des nodules (Tableau 3) a été influencée par l'apport de Phosphore. Birot et al. (1983) ont également signalé que le nombre des nodules de Phaseolus diminuaient assez rapidement après la floraison et cela semblait en partie un effet des bactéroïdes, et en partie un effet de l'environnement nodulaire (effet de l'hôte). Ainsi, le point crucial peut être le timing du fait que 4 SAS a donné des résultats satisfaisant par rapport à 6 SAS dans les deux sites expérimentaux. Le maintien d'un niveau élevé de fixation d'azote dans la période de remplissage des gousses pourrait bien être le facteur le plus important à rechercher.

Le haricot a la capacité de fixer l'azote atmosphérique $\left(\mathrm{N}_{2}\right)$ à l'aide des bactéries symbiotiques du genre Rhizobium ou phaseoli, qui sont logées dans les nodules de leurs racines (Dilworth et al, 2008). Par contre, le sol doit contenir la bactérie appropriée à la légumineuse. Dilworth et al. (2008) mentionnent que l'espèce et le nombre de Rhizobiums retrouvés dans un sol changeaient selon la fréquence de la culture as sociée au Rhizobium ou phaseoli. Présentement, lors de semis du haricot à l'Est de la RDC, aucune inoculation des semences n'est faite. Malheureusement, lors de ces essais, aucune évaluation de la quantité et du type de Rhizobium présent dans le sol n'a aussi été faite. Par ailleurs, en présence de nitrates dans le sol, les légumineuses suspendent le processus de fixation et s'alimentent à partir de nitrates du sol comme les autres familles de plante, car ce mécanisme demande plus d'énergie (Lindermann et Glover, 1990). Lindermann et Glover (1990) mentionnent que certaines légumineuses, comme le haricot, fixeraient moins d'azote que leurs besoins. Ces faits peuvent expliquer l'augmentation des recommandations en $\mathrm{N}$ et $\mathrm{P}$ pour cette culture (Graham et al., 1979).

Le rendement en grain le plus élevé a été obtenu avec l'option GIFS 40-60 N: $\mathrm{P}_{2} \mathrm{O}_{5} \mathrm{~kg} \mathrm{ha}^{-1}$ et $5 \mathrm{t} \mathrm{ha}^{-1}$ fertilisation (1781.23 kg ha $\mathrm{kg}^{-1}$ «abare» and $1641.39 \mathrm{~kg} \mathrm{ha}^{-1}$ «Walungu»), mais n'étant pas une dose économique. Des rendements nets significativement plus élevés (1637,50 ha ${ }^{-1}$ 《Kabare » and 1641,67 kg ha

1 «Walungu») et leurs ratio bénéfice-coûts $(2,82$ «Kabare» and 2,87 «Walungu») ont été obtenus avec l'application de $60 \mathrm{P}_{2} \mathrm{O}_{5} \mathrm{~kg} \mathrm{ha}^{-1}$ dans les deux sites d'étude.

\section{Conclusion}

Le rendement maximal de la culture de haricot commun est un corollaire de l'application d'engrais à leurs optima. La présente étude a été lancée pour évaluer l'influence de $\mathrm{N}$ et $\mathrm{P}$ sur croissance, la nodulation et le rendement ainsi que la rentabilité économique du haricot commun. Les propriétés physiques et chimiques du sol ont été recueillies avant la plantation. Des rendements nets significativement plus élevés ont été obtenus 
avec l'application de phosphore $60 \mathrm{P}_{2} \mathrm{O}_{5} \mathrm{~kg} \mathrm{ha}^{-1}$ suivi d'azote $40 \mathrm{~N} \mathrm{~kg} \mathrm{ha}^{-1}$ dans les deux sites d'étude du fait que les cultivars de haricots présentent différents modèles d'accumulation de $\mathrm{N}$ et de $\mathrm{P}$, en fonction de du phosphore natif du sol. Les réponses de la croissance végétative à un apport plus élevé en $\mathrm{P}$ ne sont pas directement corrélées avec la même le rendement en graines mais seulement avec la nodulation. Un apport en $\mathrm{N}$ sous-optimal peut limiter l'expression du potentiel de rendement des cultivars, réduisant ainsi la variabilité génotypique des réponses de $\mathrm{P}$. De manière générale, il a recommandé que d'autres études soient menées sur d'autres sites de Walungu et Kabare afin d'extrapoler nos résultats sur tous les territoires de notre étude. De plus, il est important de connaître la teneur en minéraux du sol qui empêche l'absorption du phosphore par la plante.

\section{Remerciements}

Nous remercions le centre CIDEP pour son accompagnement dans l'accomplissement de notre recherche et aussi les auteurs des universités partenaires et le CIAT HarvestPlus à travers le programme de coopération avec ULGL pour leur appui scientifique.

\section{Références bibliographiques}

[1] Allen, D.J., Ampofo, K.A. and Wortmann, C.S. 1997. Field Problems of beans in Africa. CITA and Centre for Tropical Agriculture (CTA). International Lvestock Research Institute (ILRI), Addis Ababa.

[2] Bado, B.V. 2002. Rôle des légumineuses sur la fertilité des sols ferrugineux tropicaux des zones guinéennes et soudaniennes du Burkina Faso. Thèse de Doctorat $(\mathrm{PhD})$ Département des sols et Environnement, Université Laval, Québec, Canada.

[3] Bennema, J. 1977. Soils. In ecophysiology of tropical crops, Alvin, P.T. and Kozlowski (eds.). Academic Press New York.

[4] Birot, A. M., Trinchant, J. C. and Rigaud, J. 1983. Nitrogen fixation in French-bean nodules in relation to aging: role of bacteroids. Physiol. V6g. 21:'115-722.

[5] Bouyoucos, G.J. 1962. Hydrometer Method Improved for Making Particle-Size Analysis of Soils. Agronomy Journal, 54, 464-465.

[6] Veeresh, N.K., 2003. Response of French bean (Phaseolus vulgaris L.) to fertilizer levels in Northern Transitional Zone of Karnataka. M.Sc. (Agri.) Thesis, Univ. Agric. Sci., Dharwad (India).

[7] Noronha, R, 1987. The World Bank Research Observer, Volume 2, Issue 2, 1 July 1987, Pages $143-$ 169, https://doi.org/10.1093/wbro/2.2.143.

[8] Filho, J.G.S., Rabbani, A.R.C., Silva, T.R.S., Silva, A.V.C., Souza, I.A., AraujoSantos, M.J.B., Jesus, J.R., Nogueira, P.L., Duringer, J.M., 2012. Chemical and molecular characterization of fifteen species from the Lantana (Verbenaceae) genus. Biochemical Systematics and Ecology, Volume 2, Issue 2, 1 July 2012, Pages 130-137, https://doi.org/10.1016/j.bse.2012.07.024

[9] Lindemann, W., and Glover, C. 1990. Nitrogen Fixation by Legumes. Retrieved March 15, 2015, from http://www.csun.edu/ hcbio027/biotechnology/lec10/lindemann.html

[10] Thirumalai, M. and Abdul Khalak, 1993. Fertilizer application economics in French bean. Curr. Res., 22: 67-69.

[11] Black, C.A. 1986. Methods of soil analysis. Part I. Physical and mineralogical properties including statistics of measurement and samplings Part II. Chemical and Microbiological properties. Agronomy series. ASA. Madison. Wis. USA. Bray, R. H. and L. T. Kurtz (1945). Determination of total organic and available forms of phosphorus in soils. Soil Science. 59:39-45.

[12] Gomez K.A. and Gomez, A.A., 1984. Statistical Procedures for Agricultural Research, 2nd edition, A wiley Inter Science Publication, New York (USA), p. 680.

[13] Gupta, P.K., Kalyan singh, Singh, U.N., Singh, R.N. and Bohra, J.S., 1996, Effect of moisture regime and fertility level on growth, yield, nutrient turnover and moisture use by French bean (Phaseolus vulgaris). Indian J. Agric. Sci., 66: 343-347.

[14] Jagdale, R.B., Khawale,V.S., Baviskar,P.K., Doshinge, B.B. and Kore, M.S., 2005. Efect of inorganic and organic nutrients on growth and yield of French bean (Phaseolus vulgaris L.). J. Soil Crops, 15: 401-405. 
[15] Kumar, M., Sinha, K.K. and Roy Sharma, R.P., 2004. Effect of organic manure, NPK and boron application on the productivity of French bean in sandy loam soil of north Bihar. Indian J. Pulses Res., $17: 42-44$.

[16] Brady, N.C. 1990. The nature and properties of soils. Macmillan, New York.

[17] Brucher H., Trop., Nutzpfl. Springer V. and Wolf G. (1977). Distribution, Yield and Use of Common Bean.

[18] Chakirwa, Z.P., Sarkodie-Addo, J. and Adjei-Gyapong T. (2017). Response of Cowpea to Zinc fertilizer application in semi-deciduous Ghana Forest, (KNUST), Kumasi, Ghana, Thesis; 1 - 116.

[19] Chakirwa, Z.P., Lubobo, A.K. and Walangululu, M.J. (2011). Field legume response to ISFM technology (minerals and organic fertilizer) in South-Kivu (Walungu), UCB, DR Congo; Thesis 1 76.

[20] Craswell, E.T., De Datta, S. K., Obcemea, W.N. and Hartantyo, M. (1981). Time and mode and nitrogen fertilizer application to tropical wetland rice. IRRI. Fertilizer research 2: 247 - 259.

[21] Eck, V. 1976. Van Eck W.H. Ultrastructure of forming and dormant chlamydospores of Fusarium solani in soil. Canadian Journal of Microbiology, 22 (1976), pp.

[22] Graham, P. H. and Rosm, J. C. (1979). Phosphorus fertilization and symbiotic nitrogen fixation in common bean. Agron. 1. 71:925-926.

[23] Greenland, 1982. Characterization of soils in relation to their classification and management for crop production: examples from some areas of the humid tropics. Clarendon Press. Oxford.

[24] Harper, J. E. 1974. Soil and symbiotic nitrogen requirements for optimum soybean production. Croo Sci. 14 255-260.

[25] International Crop for Research In Semi-Arid Tropics (ICRISAT). 1991. Phosphorous nutrition of grain legumes in the semi-arid tropics. Johansen, K.K. and K.L. Sahrawat (eds.).

[26] International Rice Research Institute. 1990. Phosphorus requirements for sustainable agriculture in Asia and Oceania. Proceedings, of a symposium 6-10th March 1989.

[27] Ladha, J. K., Pathak H., Krupnik, T. J, Six., J, van Kessel, C. 2005. Efficiency of fertilizer nitrogen in cereal production: retrospect and prospects. Advances in Agronomy 87: 85 - 156.

[28] Maiti, R.K. and Singh, V.P. 2007. Advances in Common Bean and Related Species, 1st edit. Publisher: Agro bios (International). Pp 1-2. Plant Physiology. 84: 835-840.

[29] Nelson, D.W. and L.E. Sommers, 1982. Total carbon, organic carbon and organic matter: In: A.L. Page, R.H. Miller and D.R. Keeney) Methods of soil analysis.

[30] Nyandat, N.N. and Michieka, D.O. 1970. Soils of Kirima Kimwe, Faculty of Agriculture Farm, National Agricultural Laboratories, Ministry of Agriculture, Kenya: 1-2.

[31] Page, A. L., R. H. Miller and D. R. Keeney 1982. Methods of soil analysis; 2. Chemical and microbiological properties, 2. Aufl. 1184 S., American Soc. of Agronomy (Publ.), Madison, Wisconsin, USA, gebunden 36 Dollar.

[32] Rowland, J.R.J. 1993. Dryland farming in Africa. The Macmillan Press Ltd London.

[33] Soils laboratory staff, 1994. Soil Laboratory Analysis. USA, Etat-Unis

[34] Ssali, H. and Keya, S. O. 1983. The effect of phosphorus on nodulation, growth and dinitrogen fixation by beans. Biol. Agric. Hortic. 1:135-144.

[35] Tisdale, S.L., Nelson W.L., and daisies D.B. 1990. Soil fertility and fertilizers. Macmillan publishing company, New York.

[36] Weiss, E.A. 1983. Oilseed Crops. Longman, New York, USA.

[37] Devlin, R.M. and Witham, F.G. 1983. Plant physiology (4th ed.). CBS, New Delhi, India. 